\title{
CONOCIMIENTO ACTUAL DE LOS ENEMIGOS NATURALES (HYMENOPTERA: APHELINIDAE) DE ESCAMAS ARMADAS (HEMIPTERA: DIASPIDIDAE) EN MÉXICO Y DESCRIPCIÓN DE UNA ESPECIE NUEVA DE COCCOBIUS
}

\author{
Svetlana NiKolaevna MYARTSEVA, Enrique RUIZ-CANCINO \& Juana María CORONADO-BLANCO* \\ Facultad de Ingeniería y Ciencias, Universidad Autónoma de Tamaulipas, Ciudad Victoria, 87149, Tamaulipas, \\ México. \\ *Autora de correspondencia: <jmcoronado@uat.edu.mx> \\ Recibido: 08/06/2015; aceptado: 03/12/2015
}

Myartseva, S. N., Ruiz-Cancino, E. \& Coronado-Blanco, J. M. 2016. Conocimiento actual de los enemigos naturales (Hymenoptera: Aphelinidae) de escamas armadas (Hemiptera: Diaspididae) en México y descripción de una especie nueva de Coccobius. Acta Zoológica Mexicana (n. s.), 32(1): 81-89.

RESUMEN. Algunas especies de la familia Aphelinidae son los parasitoides más importantes de las escamas armadas (Hemiptera: Diaspididae) de importancia económica en el mundo. Actualmente se han identificado 44 especies en cinco géneros como parasitoides de 27 especies de escamas armadas en México. En el presente escrito se incluye información sobre los géneros de afelínidos que atacan escamas armadas en el país, se elaboró la clave para identificación de géneros de Aphelinidae parasitoides de Diaspididae en México, así como listas de afelínidos parasitoides y sus huéspedes. Adicionalmente, se describe una especie nueva del género Coccobius Ratzeburg que parasita Diaspididae.

Palabras clave: Aphelinidae, Diaspididae, nueva especie, Coccobius, México.

\section{INTRODUCCIÓN}

Las escamas armadas (Hemiptera: Diaspididae) son insectos fitófagos que pueden ser plagas importantes en cítricos y otros frutales, ornamentales y en árboles forestales. La fauna mundial de Diaspididae incluye 199 especies plaga de diversas plantas y 43 de ellas son plagas primarias (Kosztarab 1990). Tradicionalmente, en esta familia se encuentra el mayor número de especies que son plagas en cítricos, situación ya reportada desde hace más de medio siglo por Ebeling (1959).

En México se han registrado 224 especies de 68 géneros de Diaspididae (Miller 1996) y 41 de ellas se reportan como plagas de importancia económica (Gaona-García 2004) incluyendo Chrysomphalus aonidum (L.), Hemiberlesia lataniae (Signoret), Comstockaspis perniciosa Comst. [= Quadraspidiotus perniciosus], Aonidiella aurantii Maskell y Lepidosaphes beckii (Newman) que cau-
Myartseva, S. N., Ruiz-Cancino, E. \& Coronado-Blanco, J. M. 2016. Current knowledge of natural enemies (Hymenoptera: Aphelinidae) of armoured scales (Hemiptera: Diaspididae) in Mexico and description of a Coccobius new species. Acta Zoológica Mexicana (n. s.), 32(1): 81-89.

ABSTRACT. Some species of the family Aphelinidae are the most important parasitoids of armored scales (Hemiptera: Diaspididae) economically important in the world. At present, 44 species in five genera had been identified as parasitoids of 27 species of armored scales in Mexico. Information about the genera of Aphelinidae attacking armored scales in this country is included in this manuscript, a key for their identification, and complete lists of aphelinid parasitoids and their hosts are composed. Moreover, a new species of the genus Coccobius Ratzeburg, parasitoid of Diaspididae, is described.

Key words: Aphelinidae, Diaspididae, new species, Coccobius, Mexico.

san más daño a plantas frutales, ornamentales y de sombra (Correa 1989, Gaona-García 2004).

La familia Aphelinidae (Hymenoptera: Chalcidoidea) contiene en México 206 especies de 14 géneros (Myartseva et al. 2012, 2013, 2014). Los afelínidos son parasitoides primarios o hiperparasitoides de diversos insectos, principalmente del orden Hemiptera. Muchas especies tienen gran importancia económica como agentes de control biológico y en el manejo integrado de plagas, generalmente contra especies de Aleyrodoidea y Coccoidea (Noyes 2014). Conviene señalar que se han introducido en los últimos 50 años algunas especies de Aphelinidae de los géneros Aphytis, Encarsia y Pteroptrix a México, contra especies de diaspídidos y aleyródidos plaga de los cítricos (Ruiz et al. 2006, Myartseva et al. 2007, 2008, 2012).

En este artículo se presentan datos sobre los enemigos naturales (Aphelinidae) de escamas armadas en México, 
se da una lista de las especies de parasitoides identificadas y sus huéspedes; además se presenta una clave para la identificación de géneros de parasitoides y la descripción de una especie nueva de Coccobius obtenida de Diaspididae en San Luis Potosí, México.

\section{MATERIAL Y MÉTODOS}

Se utilizaron materiales depositados en el Museo de Insectos de la Facultad de Ingeniería y Ciencias de la Universidad Autónoma de Tamaulipas (UAT), en Cd. Victoria, Tamaulipas, que han sido colectados por los autores en los últimos 15 años. La colección cuenta con más de 1,200 montajes de Aphelinidae en preparaciones microscópicas. Las especies fueron identificadas con claves publicadas por especialistas en Aphelinidae (Hayat \& Khan 2010, Myartseva 2000). Los nombres de las especies de Diaspididae se anotaron de acuerdo con la nueva información taxonómica de ScaleNet (Ben-Dov 2001).

\section{RESULTADOS Y DISCUSIÓN}

Según los datos de la colecta del material examinado y el análisis de las publicaciones, en México se han identificado 44 especies de cinco géneros de Aphelinidae como parasitoides de 27 especies de Diaspididae (Cuadros $1 \mathrm{y}$ 2), excluyendo los dos géneros hiperparasitoides, además se han recolectado 12 especies de Aphelinidae que han emergido de Diaspididae no determinados.

La mayor parte de los registros en México corresponden a escamas armadas de cítricos como la escama roja de California Aonidiella aurantii, escama roja de Florida Chrysomphalus aonidum, escama púrpura Lepidosaphes beckii, escama guante Lepidosaphes gloverii, escama paja Parlatoria pergandii Comstock, escama nieve de los cítricos Unaspis citri (Comstock), escama nieve menor Pinnaspis strachani Cooley y la escama amarilla Aonidiella citrina (Ruiz et al. 2006, Varela-Fuentes et al. 2013). Otros registros sobre frutales y plantas ornamentales que son atacados por las escamas armadas incluyen a Hemiberlesia cyanophylli (Signoret), Comstockaspis perniciosa y Pinnaspis strachani. Cabe aclarar que algunas de las especies que viven en cítricos usualmente también están presentes en otros frutales u ornamentales (Gaona-García 2004).

Las escamas armadas donde se registró un mayor número de parasitoides son las más polífagas como $A$. $a u$ - rantii (11 especies de parasitoides), C. aonidum (cinco especies), Diaspis echinocacti (cinco especies), C. perniciosa (cuatro especies) y U. citri (cinco especies).

Generalmente estos afelínidos tienen de una a dos especies de escamas armadas como huéspedes, aunque seis especies de estos parasitoides tienen a tres especies de escamas armadas como huéspedes. La especie más polífaga es Encarsia citrina, la cual se ha obtenido de nueve especies de escamas armadas en México, mientras que Aphytis lingnanensis ha emergido de cuatro especies de escamas armadas (Cuadro 2).

Antes del presente trabajo, en México se conocían 24 especies de parasitoides de escamas armadas de tres géneros de Aphelinidae (Aphytis-18, Encarsia-5, Pteroptrix1) (Myartseva \& Ruiz-Cancino, 2000) y como resultado de la presente revisión, la lista de parasitoides primarios incluye 44 especies de cinco géneros (Aphytis-19, Coccobius-3, Encarsia-17, Neophytis-2, Pteroptrix-3), excluyendo Ablerus y Marietta que son hiperparasitoides (Cuadro 2).

De las 44 especies de Aphelinidae que son parasitoides de Diaspididae en México, 18 especies nuevas de cuatro géneros fueron descritas por los presentes autores en la última década. A continuación se presenta la clave para los géneros de Aphelinidae que atacan escamas armadas en México.

\section{Clave para identificación de géneros de Aphelinidae parasitoides de Diaspididae en México}

1. Todos los tarsos de cuatro segmentos ................... Pteroptrix Westwood - Todos los tarsos de cinco segmentos o sólo los tarsos medios de cuatro segmentos . . . . . . . . . . . . . 2 2. Antena cuando mucho con seis segmentos; maza no segmentada. Alas anteriores con línea calva. ........ 3 - Antena al menos con siete segmentos; maza con dos o tres segmentos. Alas anteriores sin línea calva....... .5 3. Propodeo cuando mucho $1 \mathrm{x}$ tan largo como el metanoto; margen posterior del propodeo nunca con crénulas; ala anterior usualmente con patrones moteados formados por la membrana oscura .......... Marietta Motschulsky - Propodeo más de dos veces tan largo como el metanoto; margen posterior del propodeo siempre con crénulas; ala anterior a veces con patrones moteados formados por setas oscuras . . .................... 4 4. Axila con seta; maza antenal sin una sensila especial gruesa en el ápice; sutura entre la maza y el segmento funicular 3 usualmente distinguible .... Aphytis Howard - Axila sin seta; maza antenal con una sensi- 
Cuadro 1. Especies de Diaspididae y sus parasitoides Aphelinidae en México.

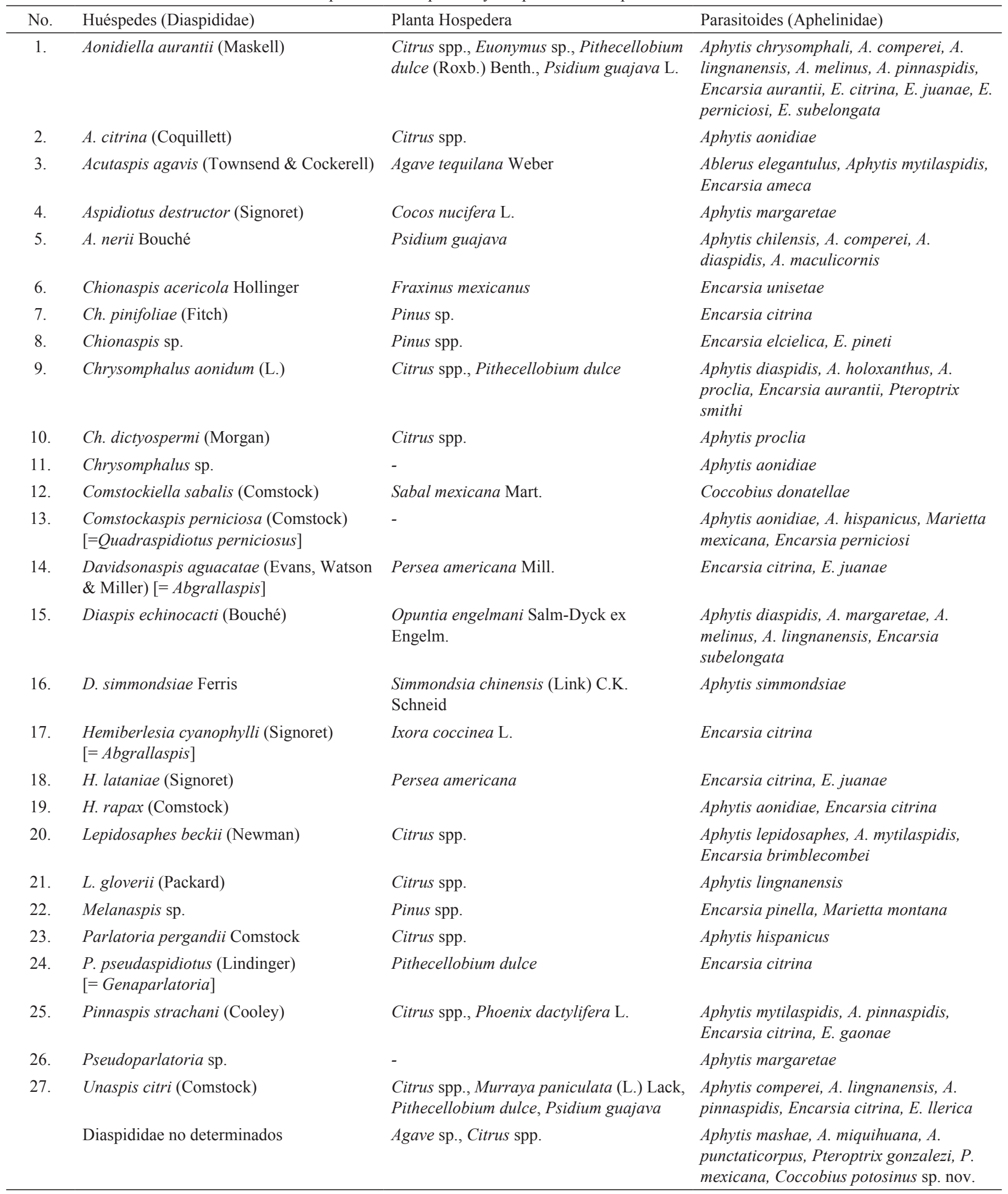


Myartseva et al.: Enemigos naturales de escamas armadas en México

Cuadro 2. Aphelinidae parasitoides primarios y secundarios de escamas armadas y sus huéspedes en México.

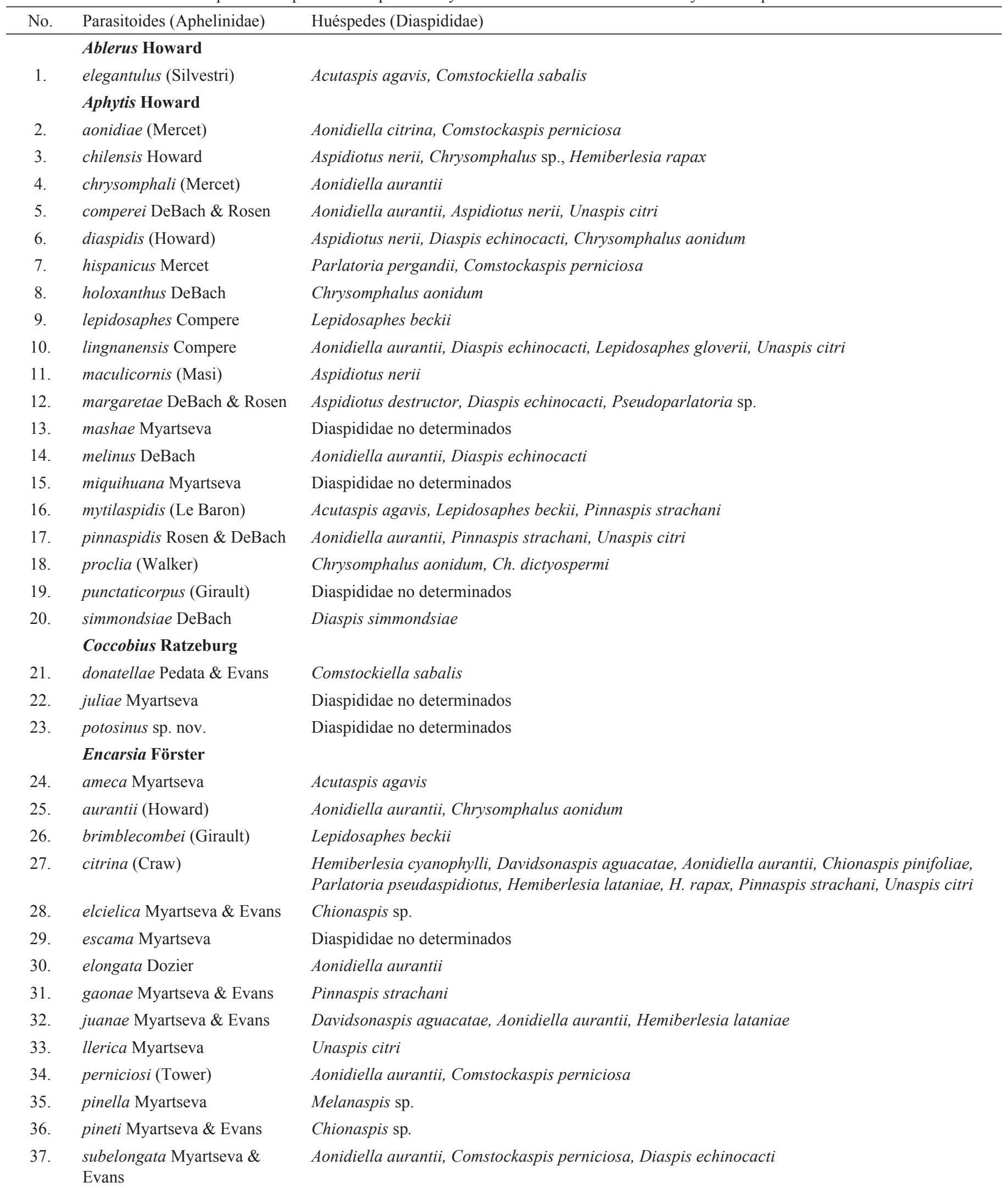


Cuadro 2. Continúa.

\begin{tabular}{|c|c|c|}
\hline 38. & unisetae Myartseva \& Evans & Chionaspis acericola \\
\hline 39. & chichenitza Myartseva & Diaspididae no determinados \\
\hline \multirow[t]{2}{*}{40.} & oaxaca Myartseva & Diaspididae no determinados \\
\hline & Marietta Motschulsky & \\
\hline 41. & mexicana (Howard) & Comstockaspis perniciosa \\
\hline \multirow[t]{2}{*}{42.} & $\begin{array}{l}\text { montana Myartseva \& } \\
\text { Ruiz-Cancino }\end{array}$ & Melanaspis sp. \\
\hline & Neophytis Kim \& Heraty & \\
\hline 43. & melanostictus (Compere) & Diaspididae no determinados \\
\hline \multirow[t]{2}{*}{44.} & myartsevae Kim \& Heraty & Diaspididae no determinados \\
\hline & Pteroptrix Westwood & \\
\hline 45. & gonzalezi Myartseva & Diaspididae no determinados \\
\hline 46. & mexicana Myartseva & Diaspididae no determinados \\
\hline 47. & smithi (Compere) & Chrysomphalus aonidum \\
\hline
\end{tabular}

la especial gruesa en el ápice; sutura entre la maza y el segmento funicular 3 usualmente no distinguible . . . . . . . . . . . . . . Neophytis Kim \& Heraty 5. Axilas pequeñas, apenas proyectadas hacia delante y ampliamente separadas. Antena de siete segmen-

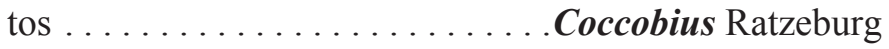
- Axilas grandes, claramente proyectadas hacia delante y separadas por no más de la longitud de una axila. Antena de siete $\mathrm{u}$ ocho segmentos. . . . . . . . . . . 6 6. Alas anteriores usualmente con patrones o bandas hialinas y ahumadas. Vena estigmal con ápice bien expandido. Pronoto entero. . . . . . . . . . . . . Ablerus Howard - Alas anteriores usualmente sin patrones ni bandas hialinas y ahumadas. Vena estigmal usualmente sin ápice bien expandido. Pronoto no entero..... . . Encarsia Förster

\section{Géneros de Aphelinidae de México parasitoides de Diaspididae}

\section{Ablerus Howard}

Género con 90 especies (Noyes 2014). Todas las especies son hiperparasitoides de diversos Hymenoptera que son parasitoides primarios. En México sólo se ha obtenido $A$. elegantulus de dos especies de Diaspididae - Comstockiella sabalis y Acutaspis agavis, por lo que requiere de más estudios taxonómicos y biológicos en el país.

\section{Aphytis Howard}

De distribución mundial consiste de 109 especies (Noyes 2014). En México se han registrado 19 especies (Myartseva et al. 2010,2012). Todas son parasitoides primarios de escamas armadas, incluyendo varias plagas primarias de Citrus spp., frutales y plantas ornamentales (Cuadro 1). Las especies reportadas para México, fueron obtenidas de 27 especies de diaspídidos.

Aphytis lingnanensis se ha encontrado en cuatro huéspedes: Aonidiella aurantii, Lepidosaphes gloverii, Unaspis citri y Diaspis echinocacti. Por su parte, Aphytis chilensis, A. comperei, A. diaspidis, A. margaretae y A. pinnaspidis tienen tres hospederos cada una. A. aonidiae, A. hispanicus, A. melinus, A. mytilaspidis y A. proclia se han obtenido de dos especies diaspídidas, por lo que 11 especies de parasitoides son de hábitos polífagos. Para las otras ocho especies se conoce sólo un hospedero para cada una. Siete especies de Aphytis fueron introducidas a México para el control biológico de escamas armadas, principalmente en Citrus spp., éstas son: A. chilensis, $A$. chrysomphali, A. hispanicus, A. holoxanthus, A. lepidosaphes, A. lingnanensis y A. maculicornis (Myartseva \& Ruiz-Cancino 2000).

El primer estudio sistemático de Aphytis fue elaborado por Compere (1955). Rosen y DeBach (1979) publicaron una monografía de la fauna mundial de Aphytis. En la lista comentada de Aphelinidae de México se incluyeron 18 especies, siguiendo a Rosen \& DeBach (Myartseva \& Ruiz-Cancino 2000). La clave para las especies de Aphytis de México, con comentarios, fue preparada por Myartseva et al. (2010). Actualmente en México se conocen 19 especies, incluyendo dos descritas de Tamaulipas ( $A$. mashae and A. miquihuana) ya que A. melanostictus fue excluída del género y transferida a Neophytis, un género recientemente descrito por Kim y Heraty (2012). En el 
Museo de Insectos de la FIC-UAT se encuentran materiales mexicanos de Aphytis comperei, A. holoxanthus, $A$. lingnanensis y A. pinnaspidis.

\section{Coccobius Ratzeburg}

Género que incluye 94 especies (Noyes 2014), todas son parasitoides primarios de Diaspididae. Para Mexico se reportaron tres especies (Myartseva 2000). Sin embargo, al estudiar nuevamente el material obtenido de Diaspididae en el Estado San Luis Potosí que había sido identificado erróneamente como $C$. stanfordi Howard, se encontró que era una nueva especie, $C$. potosinus sp. nov., la cual se describe en este artículo.

\section{Coccobius potosinus Myartseva, sp. nov.}

Material. Holotipo hembra: México, San Luis Potosí, ex Diaspididae no determinados, 12.XI.1999 (col. S.N. Myartseva). [Identificación errónea como Coccobius stanfordi en Myartseva, 2000]. Holotipo depositado en el Entomological Research Museum, University of California-Riverside, USA (UCRC).

Hembra. Longitud del cuerpo: $1.10 \mathrm{~mm}$.

Coloración. Cabeza negra. Antena con radícula, escapo $\mathrm{y}$ el segmento funicular 1 negro parduzcos; pedicelo $\mathrm{y}$ segmentos funiculares 2 y 3 blancuzcos; maza amarillo parduzco. Mesosoma negro parduzco, metanoto medialmente y lóbulos laterales basalmente amarillentos. Alas anteriores hialinas. Coxas negras; coxa media en el tercio apical y coxa posterior apicalmente amarillo blancuzcas; fémures negros; tibias principalmente negras, el tercio apical de las tibias frontales y medias y la porción apical de las tibias medias son blanco amarillento; tarsos blancuzcos y con el segmento apical ahumado. Gáster negro parduzco; terguitos 1- 3 medialmente blanco amarillentos; valvífer 2 amarillo.

Estructura. Cabeza tan ancha como el mesosoma, ligeramente más ancha que alta. Frontovértice 0.5 veces tan ancho como la anchura de la cabeza, con escultura transversal elongada reticulada y setas erectas. Distancia entre el ocelo posterior y el ojo tan larga como el diámetro de un ocelo. Ojos 1.6 veces tan largos como las mejillas. Antenas (Fig. 1) insertadas inmediatamente debajo del nivel del margen inferior de los ojos. Distancia entre tórulos más o menos tan larga como la distancia y aproximadamente 0.6 veces tan larga como la distancia al ojo. Antena con radícula 2.5 veces tan larga como ancha; escapo 3.5
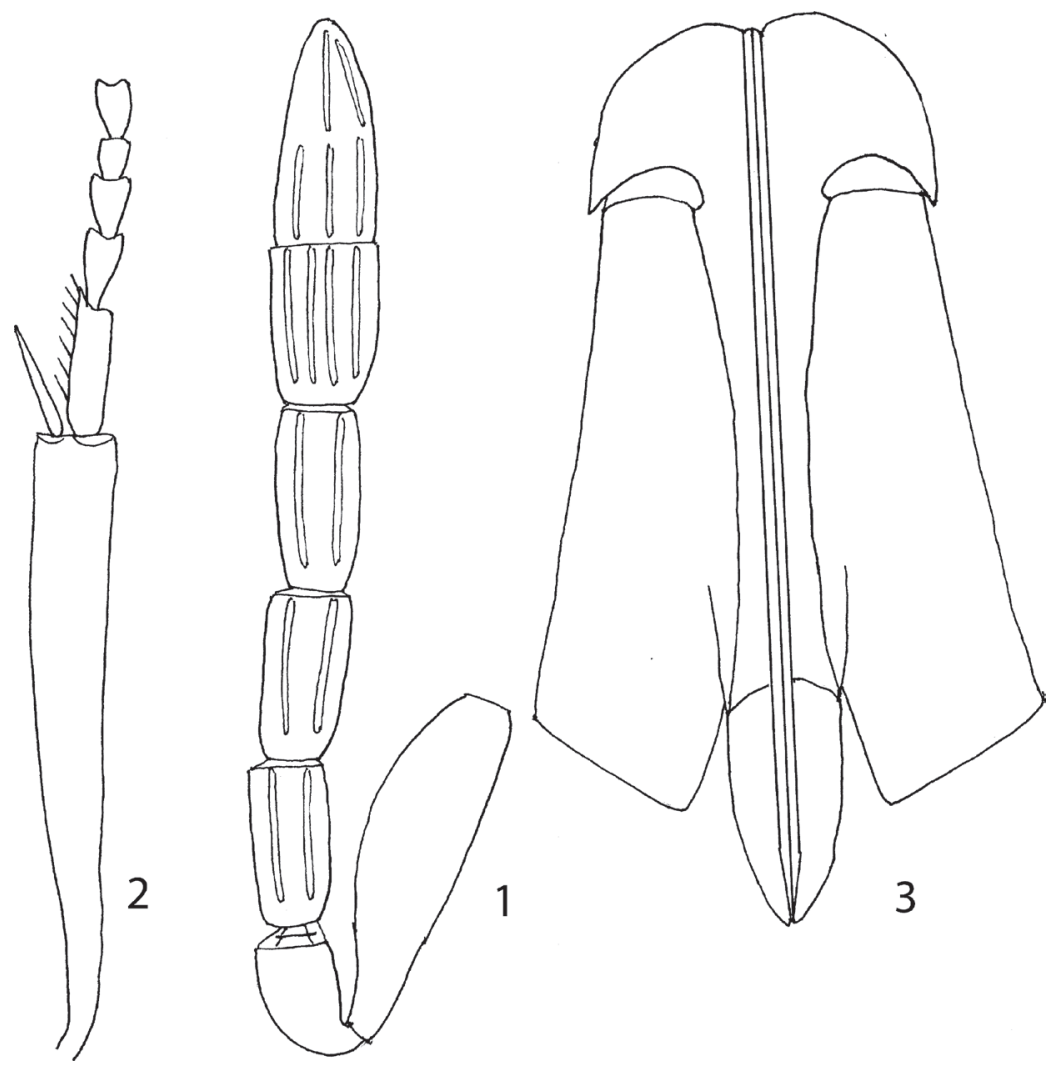

Figuras 1-3. Coccobius potosinus sp. nov., hembra: 1-antena, 2-tibia y tarso medio, 3-ovipositor (todas x 200). 
veces tan largo como ancho; pedicelo más o menos 1.4 veces tan largo como ancho y visiblemente más corto que el segmento funicular 1, el cual es aproximadamente 2 veces tan largo como ancho; segmento 2 un poco más largo y más ancho, 1.7 veces tan largo como ancho; segmento 3 un poco más largo y 1.8 veces tan largo como ancho; maza con el segmento 1 más o menos tan largo como el segmento funicular precedente pero más ancho y 1.3 veces tan largo como ancho, segmento apical 1.5 veces tan largo como el basal; segmentos funiculares con 2 sensilas longitudinales cada uno; segmentos de la maza con 4 sensilas cada uno. Lóbulo medio del mesoescudo con muchas setas delgadas, dos setas en el margen basal, más largas y fuertes. Escultura fina celular reticulada, puede ser vista a mayor magnificación. Escutelo ligeramente más corto que el mesoescudo con 6 setas, su escultura longitudinalmente estriada. Axila con una seta, lóbulo lateral con 2 setas. Ala anterior 2.5 veces tan larga como ancha, su fleco marginal 0.1 veces tan largo como la anchura alar; vena submarginal con 10 setas a lo largo de su margen anterior; vena marginal ligeramente más larga y con 13 setas a lo largo de su margen anterior; vena postmarginal ausente. Espuela de la tibia media (Fig. 2) ligeramente más corta que el basitarso, el cual es tan largo como los dos segmentos tarsales siguientes juntos. Ovipositor (Fig. 3) ligeramente expuesto, 1.4 veces tan largo como la tibia media; válvula 3 es 0.3 veces tan larga como el valvífer 2 y 1.3 veces tan larga como el basitarso de la pata media. Macho desconocido.

Etimología. El nombre de la especie se deriva del Estado de San Luis Potosí, donde ésta fue colectada.

Comentarios. Coccobius potosinus sp. nov., fue identificada erróneamente como C. stanfordi (Howard), una especie distribuida en Estados Unidos (Myartseva, 2000). En la clave de Hayat (1984), la nueva especie queda como
C. stanfordi mientras que en la clave de Hayat \& Khan (2010) llega a la especie oriental C. reticulatus (Compere \& Annecke). Ambas pueden separarse de la nueva especie por las características del escapo, patas y alas que se describen en el Cuadro 3.

\section{Encarsia Förster}

Este género megadiverso tiene distribución cosmopolita, es el más diverso en la familia Aphelinidae e incluso en la superfamilia Chalcidoidea, consiste de 429 especies (Noyes 2014). Parasitan huéspedes de varios órdenes de Insecta pero preferentemente hemípteros de la superfamilia Aleyrodoidea (familia Aleyrodidae) o de Coccoidea (familias Diaspididae y Coccidae). En México, Encarsia también es el género con mayor diversidad en Aphelinidae, ya que al presente se conocen 95 especies (Myartseva \& Evans 2008, Myartseva et al. 2008, 2012, 2013, 2014.). Antes se conocían sólo cinco especies de Encarsia como parasitoides de escamas armadas en México (Myartseva \& Ruiz-Cancino 2000); actualmente para 17 especies de Encarsia se han identificado igual número de especies huéspedes de Diaspididae y se han descrito 12 especies nuevas en la última década.

El grupo de especies de Encarsia aurantii incluye 22 que son parasitoides de escamas armadas (Myartseva et al. 2008). En México, seis especies de este grupo atacan Diaspididae, dos de dichas especies fueron introducidas para el control biológico de diaspídidos: E. aurantii para combatir la escama roja de Florida Chrysomphalus aonidum, mientras que E. perniciosi se utilizó contra la escama roja de California Aonidiella aurantii (García-Martell 1973). Las claves para este grupo de especies en México fueron publicadas por Myartseva et al. (2008).

En el género, el parasitoide más polífago es Encarsia citrina ( 9 especies de escamas armadas huéspedes), $E$. juanae y E. subelongata atacan tres especies de diaspídi-

Cuadro 3. Diferencias entre tres especies de Coccobius.

\begin{tabular}{llll}
\hline Características & potosinus sp. nov. & stanfordi & reticulatus \\
\hline Escapo & negro parduzco & pardo oscuro & ahumado en la base \\
Patas & principalmente negras & principalmente pardo oscuro & principalmente amarillas \\
Ala anterior & $2.5 x$ tan larga como ancha & $3.0 x$ tan larga como ancha & $2.8 x$ tan larga como ancha \\
Venas marginal y submarginal, setas & más largas y 13 setas & iguales y con 14-15 setas & más cortas y con 11 setas \\
Vena postmarginal & ausente & ausente & presente \\
O:mt* & 1.4 veces más larga & 2.0 veces más larga & $1.6-1.8$ veces más larga \\
v3:v2* & 3.0 veces más larga & 3.4 veces más larga & $2.8-3.0$ veces más larga \\
v3:mbt* & 1.3 veces más larga & 1.7 veces más larga & 1.5 veces más larga \\
\hline
\end{tabular}

*O-ovipositor, mt-tibia media, v2-valvífer 2, v3-válvula 3, mbt-basitarso de la pata media. 
dos mientras que E. aurantii y E. perniciosi parasitan dos especies de escamas armadas.

\section{Marietta Motschulsky}

El género contiene 22 especies, seis de ellas en México. La mayoría de las especies son hiperparasitoides en Hemiptera y seis han emergido de diaspídidos (Noyes 2014). Se desarrollan como ectoparasitoides de varios parasitoides primarios de Hymenoptera. Marietta montana fue obtenida de Melanaspis sp., sobre Pinus sp., en Tamaulipas, México (Myartseva \& Ruiz-Cancino 2001). Marietta mexicana es conocida como hiperparasitoide de 13 especies de Diaspididae (Noyes 2014). Hayat (1986) publicó la revisión de la fauna mundial de Marietta, con la clave para especies.

\section{Neophytis Kim \& Heraty}

Género descrito por Kim y Heraty (2012) a partir de material colectado en México. Neophytis contiene cuatro especies, incluyendo dos de México. Neophytis myartsevae Kim \& Heraty fue obtenida de un Diaspididae en Agave sp., en el Estado de Tamaulipas. Neophytis melanostictus (Compere) emergió de escamas armadas no determinadas colectadas en el Estado de Baja California Sur (Myartseva \& Ruiz-Cancino 2000). La clave para las especies del género fue preparada por Kim \& Heraty (2012).

\section{Pteroptrix Westwood}

Este género contiene 72 especies en el mundo (Noyes 2014). Todas las especies son parasitoides de Diaspididae. En México ocurren tres especies como P. smithi que fue introducida para el control de Chrysomphalus aonidum en cítricos mientras que dos especies nuevas fueron obtenidas de Diaspididae no determinados y descritas como $P$. gonzalezi de Chiapas y P. mexicana de Baja California Sur y Michoacán. La clave para la identificación de dichas especies fue publicada por Myartseva et al. (2012).

AGRADECIMIENTOS. Al proyecto PRODEP "Taxonomía y ecología de fauna y micobiota en comunidades forestales y cultivos" de la Universidad Autónoma de Tamaulipas por su apoyo. A los revisores anónimos por su contribución a la mejoría del manuscrito.

\section{LITERATURA CITADA}

Ben-Dov, Y. 2001. ScaleNet, Diaspididae. 27 June 2001. http://www. sel.barc.usda.gov/scalenet/query.htm. (Última actualización: Diciembre 2014).

Compere, H. 1955. A systematic study of the genus Aphytis Howard (Hymenoptera, Aphelinidae) with descriptions of new species. University of California Publications in Entomology, 10: 271320 .
Correa, L. A. 1989. Escamas armadas (Homoptera: Diaspididae) de frutales, pp. 117-135. En: Bravo M., H., H. González, H. \& J. López, C. (Eds.). Plagas de frutales. Colegio de Postgraduados. México. 177 pp.

Ebeling, W. 1959. Subtropical fruit pests. University of California, Berkeley, $463 \mathrm{pp}$.

Gaona-García, G. 2004. Coccoidea (Homoptera) y sus parasitoides de plantas ornamentales, frutales y de sombra de Cd. Victoria, Tamaulipas, México. Tesis de Doctorado en Ciencias. Cd. Victoria, Tamaulipas, UAT, México. 126 pp.

García-Martell, C. 1973. Primera lista de insectos entomófagos de interés agrícola en México. Fitófilo, 26(68): 1-41.

Hayat, M. 1984. Notes on some species of Coccobius and Prophyscus (Hymenoptera: Aphelinidae), with special reference to Girault and Howard types. Oriental insects, 18: 289-334.

Hayat, M. 1986. Notes on some species of Marietta (Hymenoptera: Aphelinidae), with a key to world species. Colemania, 2: 1-18.

Hayat, M. \& Khan, F. R. 2010. Additions to the Aphelinidae of India (Hymenoptera-Chalcidoidea): 1. On species of Ablerus Howard, Coccobius Ratzeburg, Coccophagus Westwood, Pteroptrix Westwood and Idiococcobius Hayat gen. nov. Colemania, 21: 1-31.

Kim, J.-W. \& Heraty, J. 2012. A phylogenetic analysis of the genera of Aphelininae (Hymenoptera: Aphelinidae), with a generic key and descriptions of new taxa. Systematic Entomology, 37: 497-549.

Kosztarab, M. 1990. Economic importance, pp. 307-311. In: Rosen, D. (Ed.). Armored scale insects. Their biology, natural enemies and control. Vol. 4 B. Elsevier, 688 pp.

Miller, D. R. 1996. Checklist of the scale insects (Coccoidea: Homoptera) of Mexico. Proceedings of the Entomological Society of Washington, 98: 68-86.

Myartseva, S. N. 2000. The genus Coccobius new for Mexico, with description of a new species and key to Nearctic species (Hymenoptera: Aphelinidae). Zoosystematica Rossica, 8: 331-334.

Myartseva, S. N. \& Evans, G. A. 2008. Genus Encarsia Förster of Mexico (Hymenoptera: Chalcidoidea: Aphelinidae). A revisión, key and description of new species. Serie Avispas Parasíticas de Plagas y Otros Insectos, 3. Universidad Autónoma de Tamaulipas, Ciudad Victoria, México. 320 pp.

Myartseva, S. N. \& Ruiz-Cancino, E. 2000. Annotated checklist of the Aphelinidae (Hymenoptera: Chalcidoidea) of Mexico. Folia Entomológica Mexicana, 109: 7-33.

Myartseva, S. N. \& Ruiz-Cancino, E. 2001. Mexican species of parasitoid wasps of the genus Marietta (Hymenoptera: Aphelinidae). Florida Entomologist, 84: 293-297.

Myartseva, S. N., Ruiz-Cancino, E. \& Coronado-Blanco, J. M. 2007. Parasitoides (Hymenoptera: Chalcidoidea: Aphelinidae) de escamas armadas (Hemiptera: Diaspididae) en Tamaulipas, México. Memorias del $19^{\circ}$ Encuentro Nacional de Investigación Científica y Tecnológica del Golfo de México, 24 y 25 de Mayo de 2007, Reynosa, Tamaulipas, México: 81-85.

Myartseva, S. N., Ruiz-Cancino, E. \& Coronado-Blanco, J. M. 2008. Encarsia aurantii species-group (Hymenoptera: Aphelinidae), parasitoids of armored scales (Hemiptera: Diaspididae) in Mexico, with key and description of a new species. Zoosystematica Rossica, 7: 67-71.

Myartseva, S. N., Ruiz Cancino, E. \& Coronado-Blanco, J. M. 2010. El género Aphytis Howard (Hymenoptera: Chalcidoidea: 
Aphelinidae) en México, clave de especies y descripción de una especie nueva. Dugesiana, 17: 81-94.

Myartseva S. N., Ruiz-Cancino E. \& Coronado-Blanco J. M. 2012. Aphelinidae (Hymenoptera: Chalcidoidea) de importancia agrícola en México. Revisión y claves. Serie Avispas parasíticas de plagas y otros insectos, no. 8. Ciudad Victoria México: Universidad Autónoma de Tamaulipas. 413 pp. [Publication on CD].

Myartseva, S. N., Ruiz-Cancino, E. \& Coronado-Blanco, J. M. 2013. Four new species of Aphelinidae (Hymenoptera: Chalcidoidea) from Mexico. Zootaxa, 3641: 223-232.

Myartseva, S. N., Ruiz-Cancino, E. \& Coronado-Blanco, J. M. 2014. Tres especies nuevas de Encarsia (Hymenoptera: Chalcidoidea: Aphelinidae) de México. Acta Zoológica Mexicana (n.s.), 30: $288-297$.
Noyes, J. S. 2014. Universal Chalcidoidea Database [online]. Worldwide Web electronic publication. www.nhm.ac.uk/entomology/ chalcidoids/index.html. (Última actualización: Agosto 2014).

Rosen, D. \& DeBach, P. 1979. Species of Aphytis of the world (Hymenoptera: Aphelinidae). Dr. W. Junk BV Publishers, London: $1-801$.

Ruiz-Cancino, E., Coronado, J. M. \& Myartseva, S. N. 2006. Situación actual del manejo de las plagas de los cítricos en Tamaulipas, México. Manejo Integrado de Plagas y Agroecología (Costa Rica), 78: 94-100.

Varela Fuentes, S. E., Orozco Santos, M., Torres Acosta, R. J. \& Silva Aguirre, J. L. 2013. Guía técnica para la identificación y manejo de plagas y enfermedades en citricos. UAT y Fundación Produce, Tamaulipas, $428 \mathrm{pp}$. 